\title{
Vitamin-D-Status entspricht der Erkrankungsaktivität im MRT
}

Fragestellung: Hat der Vitamin-D-Status bei schubförmiger Multipler Sklerose Einfluss auf die Erkrankungsaktivität, die in der Kernspintomografie quantifiziert werden kann?

Hintergrund: Seit längerem sind die Daten hinsichtlich einer möglichen Bedeutung der Vitamin-D-Serumspiegel auf die Erkrankungsaktivität bei der schubförmigen Multiplen Sklerose widersprüchlich. Allerdings deuten kleinere Fallserien sowie jetzt zwei kleinere prospektive Studien daraufhin, dass niedrige Vitamin-D-Spiegel insbesondere mit einer höheren Erkrankungsaktivität vergesellschaftet, höhere Spiegel dagegen mit einer niedrigeren Erkrankungsaktivität assoziiert sind. Auch wenn mehrere Studien inzwischen mit jeweils kleinen Fallzahlen (unter 100 Studienteilnehmern) eher für eine signifikante Rolle von Vitamin D sprechen, fehlt nach wie vor eine größere prospektive Studie, um die Hypothese zu testen.

Mowry EM, Waubant E, McCulloch CE et al. Vitamin D status predicts new brain magnetic resonance imaging activity in multiple sclerosis. Ann Neurol 2012; $72: 234-40$
Patienten und Methodik: In einer fünfjährigen longitudinalen Kohortenstudie erhielten Patienten neben einer klinischen Evaluation jährliche MRT-Aufnahmen des Kopfes sowie Blutentnah- men zur Bestimmung des 25-Hydroxy-Vitamin-D (25OH-D). Die Vitamin-D-Spiegel wurden dann hinsichtlich ihrer Assoziation mit neuen T2-gewichteten und Gadolinium aufnehmenden T1-gewichteten Herden in den Kernspintomografien ausgewertet.

Ergebnisse: Von insgesamt 469 Patienten wurden über 2.260 MRT-Aufnahmen ausgewertet. In einer multivariaten Analyse zeigte sich, dass jeder $10 \mathrm{ng} / \mathrm{ml}-\mathrm{Sch}$ ritt der $25 \mathrm{OH}-\mathrm{D}$-Spiegel mit einer 15\%igen Reduktion des Risikos neuer T2-Läsionen und mit einer 32\%igen Risikoreduktion für Gadolinium aufnehmende Läsionen vergesellschaftet war. Ein um $50 \mathrm{ng} / \mathrm{ml}$ höhere Vitamin-D-Spiegel war ferner mit einer dauerhaft niedrigeren Erkrankungsprogression assoziiert. Mit anderen Worten: Hohe Vitamin-D-Spiegel korrelierten mit einer niedrigeren (statistisch nicht signifikanten) Schubrate.

Schlussfolgerungen: Die Höhe des Vitamin-D-Spiegels ist auch in dieser Studie umgekehrt mit der in der Kernspintomografie quantifizierbaren Erkrankungsaktivität bei Patienten mit Multipler Sklerose assoziiert. Dabei scheint eine lineare Beziehung zu bestehen, die bei niedrigen Spiegeln mit einer hohen Erkrankungsaktivität und umgekehrt bei hohen Spiegeln mit einer niedrigen Aktivität assoziiert ist.

\section{-Kommentar von Volker Limmroth, Köln-Merheim}

\section{... und Vitamin D ist doch wichtig!}

Eine weitere Studie zeigt hier die nun doch immer wahrscheinlicher werdende Funktion von Vitamin D auf die Erkrankungsaktivität der Multiplen Sklerose. Aufgrund der hier deutlich größeren Studienpopulation (über 450 Patienten) und des validen Studiendesigns sind die Daten glaubhaft. Die Daten zur Erkrankungsaktivität, die kernspintomografisch gut quantifiziert werden konnten, sind recht eindeutig. Damit liegen uns allein aus den letzten sechs bis zwölf Monaten vier Studien mit etwas unterschiedlichen Schwerpunkten, aber gutem Design vor, die eine immunologische Funktion von Vitamin D stützen und einheitlich zeigen, dass höhere Spiegel mit einer Reduktion der Erkrankungsdynamik verbunden sind. Dennoch ist eine größere klinische Studie notwendig, um die These endgültig zu untermauern. Darüber hinaus ist es notwendig, möglichst schnell den - die Richtigkeit der These vorausgesetzt - Wirkmechanismus zu klären, über den Vitamin D antiinflammatorisch wirkt. Es sollten aber keine falschen Hoffnungen geweckt werden: Auch die euphorischsten bisherigen Daten deuten lediglich auf eine Wirkung in einer Größenordnung hin, die unterhalb der jetzigen immunmodulatorischen Basistherapien liegt - mehr nicht.
Festzuhalten bleibt, dass eine mögliche immunmodulatorische Therapie wahrscheinlich immer noch wirksamer ist als ein Vitamin-D-Ausgleich oder der Vergleich zwischen niedrigen und hohen Spiegeln. Dennoch scheint es inzwischen gerechtfertigt, bei ausgewählten Patienten mit schwerwiegenden Verläufen am Anfang eine Vitamin-D-Spiegelkontrolle durchführen zu lassen, um dann entsprechend besser reagieren zu können.

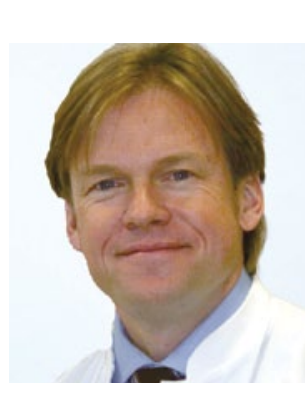

Prof. Dr. med. Volker Limmroth, Köln-Merheim

Chefarzt der Klinik für Neurologie und Palliativmedizin Köln-Merheim E-Mail: LimmrothV@kliniken-koeln.de 